\title{
Statins use and risk of new-onset diabetes
} in hypertensive patients: a population-based retrospective cohort study in Yinzhou district, Ningbo city, People's Republic of China

\author{
This article was published in the following Dove Press journal: \\ Therapeutics and Clinical Risk Management
}

\author{
Hailong $\mathrm{Li}^{\prime}$ \\ Hongbo Lin ${ }^{2}$ \\ Houyu Zhao' \\ Yang $X u^{\prime}$ \\ Yinchu Cheng' \\ Peng Shen ${ }^{2}$ \\ Siyan Zhan'
}

'Department of Epidemiology and Bio-statistics, School of Public Health, Peking University Health Science Centre, Beijing, People's Republic of China; ${ }^{2}$ Department of Chronic Diseases and Health Promotion, Yinzhou District Center for Disease Control and Prevention, Ningbo, People's Republic of China
Correspondence: Siyan Zhan Department of Epidemiology and Biostatistics, School of Public Health, Peking University Health Science Centre, 38 Xueyuan Road, Haidian District, Beijing I0019I, People's Republic of China

Tel +86 I0 82805162

Fax +86 I0 82805162

Email siyan-zhan@bjmu.edu.cn
Background: Reports have suggested that statin use is associated with an increased incidence of type 2 diabetes mellitus (T2DM). Guidelines suggested that statins should be prescribed in hypertensive patients for primary prevention. However, there were very few studies on the risk of T2DM associated with statin use among patients with hypertension in mainland People's Republic of China.

Purpose: To determine the association between statin use and new-onset diabetes mellitus among patients with hypertension in mainland People's Republic of China.

Patients and methods: We performed a retrospective cohort study of hypertensive patients using the Yinzhou regional health care database from January 1, 2010, to August 31, 2016. Patients aged 30-90 years old without T2DM were eligible for inclusion. We identified new statin initiators and nonusers by using prescription records of inpatients and outpatients. Multivariate Cox model and propensity score methods were used to adjust potential confounders, including age, sex, body mass index, comorbidities, lifestyle characteristics, and baseline antihypertensive drug use. The risk of incident T2DM among statin initiators compared to nonusers was estimated by the Cox proportional hazards model. Propensity scores for statin use were then developed using logistic regression, statin initiators were matched 1:1 with nonusers according to propensity scores with the nearest neighbor matching method within 0.2 caliper width, and Cox regression was again conducted.

Results: Among 67,993 patients (21,551 statin initiators; 46,442 nonusers), the unadjusted incidence rate of incident T2DM was higher in statin initiators than nonusers $(25.68$ versus 14.19 events/1,000 person-years; adjusted hazard ratio: 1.55 ; 95\% confidence interval: 1.44-1.66). After propensity score 1:1 matching (19,818 statin initiators; 19,818 nonusers), baseline characteristics between 2 groups were balanced except that the nonusers group was 0.53 years older on average $(P<0.001)$. Then statin use was still associated with a significant increased risk for T2DM in the matched cohort (adjusted hazard ratio: 1.54 ; $95 \%$ confidence interval: 1.41-1.67). Subgroup analyses also demonstrated similar findings.

Conclusion: Our study indicated an association between statin use and an increased risk of new-onset diabetes mellitus. It provides better understanding of statin and new-onset diabetes mellitus association among hypertensive patients in real-word setting. As an observational study, our findings were prone to unmeasured confounding and bias.

Keywords: statin, type 2 diabetes, hypertension, safety, observational study

\section{Introduction}

There is strong clinical evidence that statins can effectively reduce all-cause mortality and cardiovascular events in patients at risk of cardiovascular diseases (CVDs). ${ }^{1-3}$ 
Thus, statins are widely used for primary or secondary prevention of CVD. However, studies of statin therapy have provided conflicting results about the association between statins use and risk for incident new-onset diabetes mellitus (NOD). The first study (West of Scotland Coronary Prevention Study) that assessed this association reported a protective effect (hazard ratio [HR]: 0.70; 95\% CI: 0.50-0.98) but used nonstandardized criteria for diabetes diagnosis. However, results from subsequent hypothesis-testing trials have been inconsistent. Thus, several meta-analyses have been performed to evaluate this association. Rajpathak et $\mathrm{al}^{4}$ have conducted a meta-analysis of statins compared with placebo and included 57,593 patients from 6 trials and found an increased risk for incident diabetes mellitus (relative risk: 1.13 ; 95\% CI: $1.03-1.23$ ). Sattar et $\mathrm{al}^{5}$ reported a metaanalysis which showed statins were associated with a $9 \%$ increased risk of diabetes in 91,140 patients from 13 trials.

Based on the evidence from randomized controlled trials, ${ }^{6-9}$ systematic reviews, ${ }^{4,5}$ and a few observational studies, ${ }^{10,11}$ the United States Food and Drug Administration mandated labeling changes for all statins in February 2012. Labels now include information concerning diabetogenic effects, including inducing diabetes and increases in hemoglobin $\mathrm{A}_{1 \mathrm{c}}$ or fasting glucose level. In November 2012, the China Food and Drug Administration warned that statins users may have an increased risk of raised blood sugar levels and the development of type 2 diabetes mellitus (T2DM). Although it is based mainly on evidence from clinical trials and meta-analyses, there are limited data regarding the long-term effects of these associations in mainland People's Republic of China. Besides, several reports suggested that Chinese are more sensitive to statins at a certain dose. ${ }^{12-14}$ The guidelines on treatment and health care system are different from other countries, even Taiwan, in mainland People's Republic of China. ${ }^{15,16}$ On the other hand, hypertensive patients are at increased risk of developing diabetes mellitus. ${ }^{17,18}$ And both hypertension and diabetes are the major causes of CVD. ${ }^{19,20}$ Evidence, expert consensus, and guidelines supported that statins should be prescribed in hypertensive patients for primary prevention. ${ }^{21-23}$ However, few studies have examined whether statin use was associated with an increased incidence of T2DM in hypertensive patients. Accordingly, the objective of this study was to assess the association between statin use and NOD among patients with hypertension in mainland china.

\section{Patients and methods}

\section{Study design}

We performed a population-based retrospective cohort study among patients aged 30-90 years old with hypertension in
Yinzhou district, Ningbo city, People's Republic of China. Hypertension was defined based on the diagnosis records of outpatients or inpatients (10th revision of the International Classification of Diseases [ICD-10]: I10). Patients who did not receive statins at any time during the study were attributed to the nonusers group. Statin use was defined as statin therapy for at least 90 days during the study period (January 1, 2011, to August 31, 2016), and the date of first statin prescription was termed the index date. To avoid immortal time bias, the index date for nonusers was assigned according to the distribution of users' index date. ${ }^{24}$

We defined our diabetes endpoint as the first diagnosis of T2DM (ICD-10: E11) or a prescription for insulin or an oral antidiabetic medication as based on the data in the outpatients or inpatients records. The primary endpoint of the study was defined as the diabetes endpoint after 180 days of statin treatment during the follow-up period. We assumed that NOD within the 180 days after statin use could not be attributed to treatment. Thus, patients who had a diagnosis of diabetes within the first 180 days were censored in our time-to-event analysis. A sensitivity analysis was conducted in which all NOD cases after initiation of statin therapy are evaluated. In order to better identify the new statins users and NOD, patients were excluded from the study if they were diagnosed as having T2DM or prescribed statins before the index date.

Our study setup a look-back period (1 year preceding their index date) to define some important confounders, namely lifestyle, comorbidities, and antihypertensive drug use.

\section{Data resources}

Yinzhou is the largest district in Ningbo, an eastern coastal city of People's Republic of China, with a population of nearly 1.2 million residents. In 2005, Yinzhou Health and Family Planning Commission intended to imitate the primary care model seen in UK. They setup 287 village- or community-based health service stations. Each station has more than 2 doctors who are responsible for the primary care and chronic diseases follow-up such as hypertension, diabetes, CVD, and cancers. In the meantime, they initiated a Health Information System, which collects the health care information of the residents. By 2010, this system covered all the hospitals (3 general hospitals, 24 community health centers, and 287 community health service stations) in Yinzhou. Moreover, it integrated hospital data, public health data, and community health data which were stored in the administrative health care database and included outpatient and inpatient records, prescribing information, as well as demographic characteristics. The outpatient and inpatient 
records comprised patient ID, date of diagnosis, diagnosis name, and diagnosis code (ICD-10). Prescribing information consisted of product name, brand, generic names, dosage, and prescription date. The lifestyle characteristics were recorded using a standard questionnaire by trained staff. Before obtaining the data set, all the data from patients was anonymized and no personal details were shared with health researchers for privacy protection. This database has been used and described in a previous study. ${ }^{25-27}$ This study was approved by the Peking University Health Science Center Ethics Committee.

\section{Statistical analysis}

In baseline analysis, age and BMI are presented as mean (standard deviation) and median (interquartile range). Differences in age and BMI were analyzed by using the Student's $t$-test. Other variables are set as categorical or discrete, which were expressed as counts and percentages and evaluated by $\chi^{2}$ or Fisher's exact test between the groups as appropriate.

In the primary analysis, Cox proportional hazard regression model was used to estimate the relation between statin use and risk of NOD. We developed a multivariable model to adjust the potential confounders, including age, sex, body mass index (BMI), comorbidities (dyslipidemia, myocardial infarction, coronary artery disease, cerebrovascular accidents, heart failure, and angina pectoris), lifestyle characteristics (drinking, smoking, physical activity), and baseline antihypertensive drug use ( $\beta$-blockers, diuretics, angiotensinconverting enzyme inhibitors, angiotensin receptor blockers, calcium channel blockers).

Additionally, propensity score matching (PSM) approach was applied to balance the systematic difference between 2 groups as secondary analyses by using $\mathrm{R}$ package "MatchIt." First, a propensity score, representing the probability of being in the statin-user or non-statin user group, was calculated for each patient via a logistic regression model. Variables were selected for inclusion in the model if they were associated with both the exposure and the outcome, or associated with the outcome. Age, sex, BMI, comorbidities, lifestyles, and baseline antihypertensive drug use were finally included. Second, statin users were then 1:1 matched to nonusers according to propensity scores with the nearest neighbor matching method within 0.2 caliper width. Third, Cox model was applied again and those factors remaining imbalanced after matching would be included in the model (eg, age).

To assess the possible effect modification of variables related to both the exposure and the outcome, we carried out subgroup analyses by stratifying the study cohort according to sex (female or male), age groups $(\leq 49,50-59,60-69$, and $\geq 70$ ),
BMI categories $\left(<24,24-28\right.$ and $\left.\geq 28 \mathrm{~kg} / \mathrm{m}^{2}\right)$, smoking status (never, past, current), alcohol use (never, occasional, often), physical activity ( $<1 \mathrm{~d} / \mathrm{wk}, 1-4 \mathrm{~d} / \mathrm{wk},>4 \mathrm{~d} / \mathrm{wk}$ ), individual statins (fluvastatin, rosuvastatin, simvastatin, and atorvastatin), and potency of statins (low, moderate, and high). We defined the potency based on recommendations from the 2013 American College of Cardiology/American Heart Association guidelines (high dose: atorvastatin $\geq 40 \mathrm{mg}$ and rosuvastatin $\geq 20 \mathrm{mg}$; moderate dose: atorvastatin $10-20 \mathrm{mg}$, rosuvastatin 5-10 mg, simvastatin $20-40 \mathrm{mg}$, and fluvastatin 40-80 mg; and low dose: simvastatin $10 \mathrm{mg}$ and fluvastatin 20-40 mg). The HRs were estimated from Cox models, adjusted for age, sex, BMI, comorbidities, lifestyle, and baseline antihypertensive drug use. Age was excluded in models within age subgroups. Similarly, corresponding variables were individually excluded in models within corresponding subgroups. All tests were 2 sided, and an $\alpha$ level of 0.05 was considered statistically significant. All time-to-event analyses were performed with SAS, version 9.4 (SAS Institute Inc., Cary, NC, USA).

\section{Results}

Overall, our study identified 95,327 patients who were aged 30-90 and diagnosed as hypertension in 2010 by using the Yinzhou database (Figure 1). A total of 67,993 patients were finally enrolled after restricting the analysis to patients without missing data, newly exposed to statins, and without history of diabetes (21,551 statin users and 46,442 nonusers).

Table 1 describes the baseline characteristics of statin users and nonusers before and after PSM. In the original cohort, the statins users group and nonusers group had a significant difference in all the baseline characteristics. After PSM, baseline characteristics of two matched groups $(19,819$ statin users and 19,819 nonusers) were balanced, except that the nonusers group was a bit older. The mean age of nonusers group was 61.93 years, significantly older than statin group (61.40 years). Figure 2 shows the histograms of the density of propensity scores for original cohort and matched cohort. During the follow-up period 4,172 (6.14\%) patients developed NOD in the total cohort. According to our definition of outcome, there were 3,421 events including 1,884 events in statin group and 1,537 events among nonusers. Patients who developed NOD were older, were more overweight, and were more likely to have dyslipidemia (Table 2).

Table 3 shows the results of primary analysis for 2 different definitions of endpoints. For NOD after 180 days of statin initiation, the crude incidence of NOD per 1,000 person-years by statin exposure status was 25.68 for statin group and 14.19 for nonusers. Compared to nonusers, the crude NOD HR was 


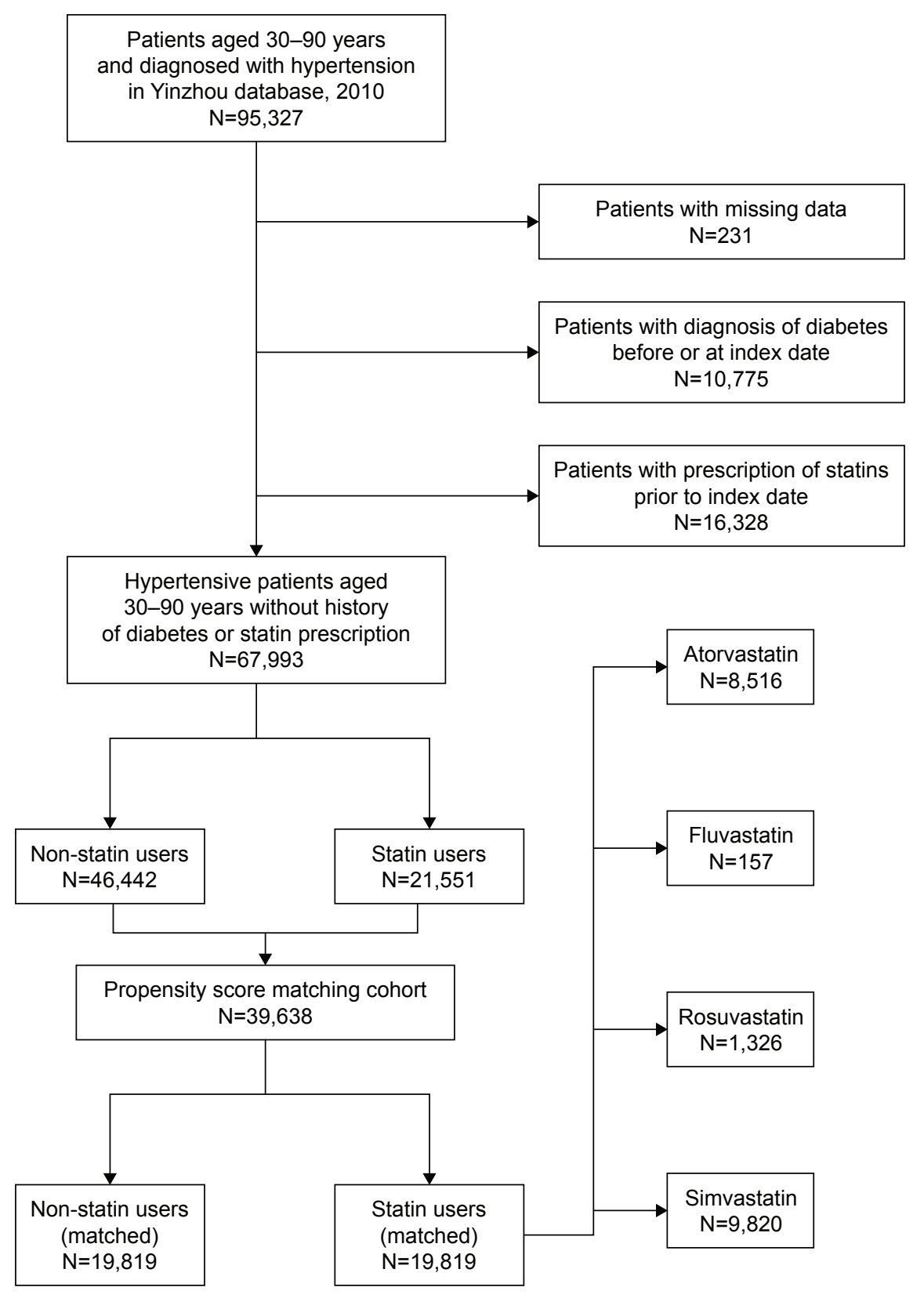

Figure I Flow chart of the study population.

1.82 (95\% CI: 1.70-1.95). Adjustments were made in Cox model for age, sex, BMI, comorbidities, lifestyle, and antihypertensive drug. The adjusted NOD HR was 1.55 (95\% CI: 1.44-1.66). After matching for propensity score and adjusting for imbalanced age, statin users showed a $54 \%$ increase in the risk for NOD with an adjusted HR of 1.54 (95\% CI: 1.41-1.67). For all NOD after statin initiation, the crude incidence of NOD in both groups was found to be higher (16.69 for statin group, 32.68 for nonusers group). The crude NOD HR was 1.96 (95\% CI: 1.84-2.08), and the adjusted NOD HR was 1.75 (95\% CI: 1.64-1.87). In the matched cohort, the adjusted NOD HR was 1.65 (95\% CI: 1.53-1.78).
As can be seen from Figure 3, the cumulative incidence of NOD was higher among new users of statins compared with non-statin users during the follow-up period.

We found consistent results in subgroup analysis assessing the association between statins use and increase incident of NOD (Table 4). Sex-specific analysis showed that the adjusted NOD HR was 1.58 (95\% CI: 1.42-1.77) among male patients, which was similar to that among female patients (adjusted HR: 1.52, 95\% CI: 1.38-1.67). In addition, age-specific HRs decreased in older people, even though the association in each stratum was statistically significant after adjustment for confounding factors. We observed 
Table I Baseline characteristics of the original and matched cohorts of hypertensive patients without history of diabetes

\begin{tabular}{|c|c|c|c|c|c|c|}
\hline \multirow[t]{2}{*}{ Characteristics } & \multicolumn{3}{|l|}{ Overall patients } & \multicolumn{3}{|l|}{ Matched patients } \\
\hline & Non-statin & Statin & $P$-value & Non-statin & Statin & $P$-value \\
\hline $\mathrm{N}(\%)$ & $46,442(100)$ & $21,551(100)$ & & $19,819(100)$ & $19,819(100)$ & \\
\hline \multicolumn{7}{|l|}{ Age } \\
\hline Mean \pm SD & $58.87(\mid 3.0)$ & $61.48(11.2)$ & $<0.001$ & $61.93(12.8)$ & $61.40(11.2)$ & $<0.001$ \\
\hline Median (QI-Q3) & $58.00(49-67)$ & $61.00(54-69)$ & & $61.00(53-72)$ & $61.00(54-69)$ & \\
\hline Sex (female) & $24,487(52.7)$ & II,989 (55.6) & $<0.001$ & I I,065 (55.8) & $10,990(55.5)$ & 0.992 \\
\hline Dyslipidemia & $\mathrm{I}, 085(2.3)$ & $2,734(12.7)$ & $<0.001$ & $\mathrm{I}, 084(5.5)$ & I,099 (5.5) & 0.982 \\
\hline MI & $13(0.0)$ & $28(0.1)$ & $<0.001$ & II (0.I) & $8(0.0)$ & 0.522 \\
\hline CAD & I,353 (2.9) & $\mathrm{I}, 2 \mathrm{I} 0(5.6)$ & $<0.001$ & $582(2.9)$ & $630(3.2)$ & 0.385 \\
\hline CVA & $428(0.9)$ & $518(2.4)$ & $<0.001$ & $247(1.2)$ & $253(1.3)$ & 0.818 \\
\hline Heart failure & $437(0.9)$ & $418(1.9)$ & $<0.001$ & $215(1.1)$ & $227(\mathrm{I} .1)$ & 0.995 \\
\hline Angina pectoris & $407(0.9)$ & $489(2.3)$ & $<0.001$ & $227(I . I)$ & $219(1.1)$ & 0.257 \\
\hline \multicolumn{7}{|l|}{ BMI, kg/m² } \\
\hline Mean \pm SD & $23.34(4.26)$ & $23.85(5.92)$ & $<0.001$ & $23.71(5.28)$ & $23.79(4.00)$ & 0.090 \\
\hline Median (QI-Q3) & $23.10(21.50-24.90)$ & $23.50(21.80-25.50)$ & & $23.50(21.60-25.40)$ & $23.50(21.80-25.50)$ & \\
\hline BMI, $\mathrm{kg} / \mathrm{m}^{2}$ & & & $<0.001$ & & & 0.811 \\
\hline$<24$ & $29,305(63.1)$ & I2,029 (55.8) & & II,07| (55.9) & II,I $50(56.3)$ & \\
\hline $24-28$ & 14,345 (30.9) & $7,642(35.5)$ & & $7,086(35.8)$ & $6,989(35.3)$ & \\
\hline$\geq 28$ & $2,792(6.0)$ & $\mathrm{I}, 880(8.7)$ & & I,662 (8.4) & I,680 (8.5) & \\
\hline Cigarette smoking & & & $<0.001$ & & & 0.421 \\
\hline Never & 34,918 (75.2) & 16,302 (75.6) & & I5,026 (75.8) & I5,003 (75.7) & \\
\hline Past & $4,156(8.9)$ & $2,268(10.5)$ & & $2,129(10.7)$ & $2,068(10.4)$ & \\
\hline Current & $7,368(15.9)$ & $2,98 \mid(13.8)$ & & $2,664(13.4)$ & $2,748(13.9)$ & \\
\hline Alcohol consumption & & & 0.024 & & & 0.952 \\
\hline Never & $36,328(78.2)$ & I6,945 (78.6) & & I5,576 (78.6) & I5,586 (78.6) & \\
\hline Occasional & $2,723(5.9)$ & $\mathrm{I}, \mathrm{I} 83(5.5)$ & & I,064 (5.4) & I,096 (5.5) & \\
\hline Often & $7,391(15.9)$ & $3,423(15.9)$ & & $3,179(16.0)$ & $3,137(15.8)$ & \\
\hline Physical activity & & & $<0.001$ & & & 0.682 \\
\hline$<\mathrm{ld} / \mathrm{wk}$ & $20,359(43.8)$ & $9,796(45.5)$ & & $9,012(45.5)$ & $8,973(45.3)$ & \\
\hline $\mathrm{I}-4 \mathrm{~d} / \mathrm{wk}$ & I5,475 (33.3) & $7,279(33.8)$ & & $6,674(33.7)$ & $6,690(33.8)$ & \\
\hline$>4 \mathrm{~d} / \mathrm{wk}$ & $10,608(22.8)$ & $4,476(20.8)$ & & $4,133(20.9)$ & $4,156(21.0)$ & \\
\hline$\beta$ blockers & $5,995(12.9)$ & $3,684(17.1)$ & $<0.001$ & $3,295(16.6)$ & $3,332(16.8)$ & 0.989 \\
\hline Diuretics & $8,654(18.6)$ & $4,982(23.1)$ & $<0.001$ & $4,577(23.1)$ & $4,523(22.8)$ & 0.659 \\
\hline ACEI & $10,277(22.1)$ & $5,780(26.8)$ & $<0.001$ & $5,310(26.8)$ & $5,244(26.5)$ & 0.407 \\
\hline ARBs & 6,863 (I4.8) & $4,300(20.0)$ & $<0.001$ & $3,900(19.7)$ & $3,934(19.8)$ & $0.88 I$ \\
\hline $\mathrm{CCBs}$ & $5,405(I 1.6)$ & 3,385 (I5.7) & $<0.001$ & $3,093(15.6)$ & $3,098(15.6)$ & 0.978 \\
\hline \multicolumn{7}{|l|}{ Type of statin } \\
\hline Non-statin & $46,442(100.0)$ & $0(0.0)$ & & $19,819(100.0)$ & $0(0.0)$ & \\
\hline Atorvastatin & $0(0.0)$ & $9,263(43.0)$ & & $0(0.0)$ & $8,516(43.0)$ & \\
\hline Fluvastatin & $0(0.0)$ & $170(0.8)$ & & $0(0.0)$ & $157(0.8)$ & \\
\hline Rosuvastatin & $0(0.0)$ & $\mathrm{I}, 403(6.5)$ & & $0(0.0)$ & $1,326(6.7)$ & \\
\hline Simvastatin & $0(0.0)$ & $10,7 \mid 5$ (49.7) & & $0(0.0)$ & $9,820(49.5)$ & \\
\hline
\end{tabular}

Note: Unless indicated all values in this table are presented as $\mathrm{n}(\%)$.

Abbreviations: MI, myocardial infarction; CAD, coronary artery disease; CVA, cerebrovascular accident; BMI, body mass index; d, day; wk, week; ACEI, angiotensinconverting enzyme inhibitors; ARBs, angiotensin receptor blockers; CCBs, calcium channel blockers.

significantly increased risk of NOD across subgroups by lifestyle (smoking, alcohol use, and physical activity). The statin-user group showed a significantly increased risk for NOD in patients with lower BMI level. Among different types of statin agents, fluvastatin had the lowest, and most statistically insignificant, risk of NOD (adjusted HR: 1.44, 95\% CI: $0.83-2.48$ ), while other three types of statins were associated with a significantly increased risk of NOD (rosuvastatin: adjusted HR: 1.47, 95\% CI: 1.19-1.83; simvastatin: adjusted
HR: $1.64,95 \%$ CI: 1.51-1.78; atorvastatin: adjusted HR: 1.52, 95\% CI: $1.37-1.68)$. Compared to nonusers, the adjusted HRs were 1.44 (95\% CI: 1.29-1.61), 1.65 (95\% CI: $1.53-1.79)$, and 1.81 (95\% CI: 0.86-2.82) for the low-, moderate-, and high-intensity statin users, respectively.

\section{Discussion}

In this population-based retrospective cohort study, we found that statin therapy was associated with an increased risk of 

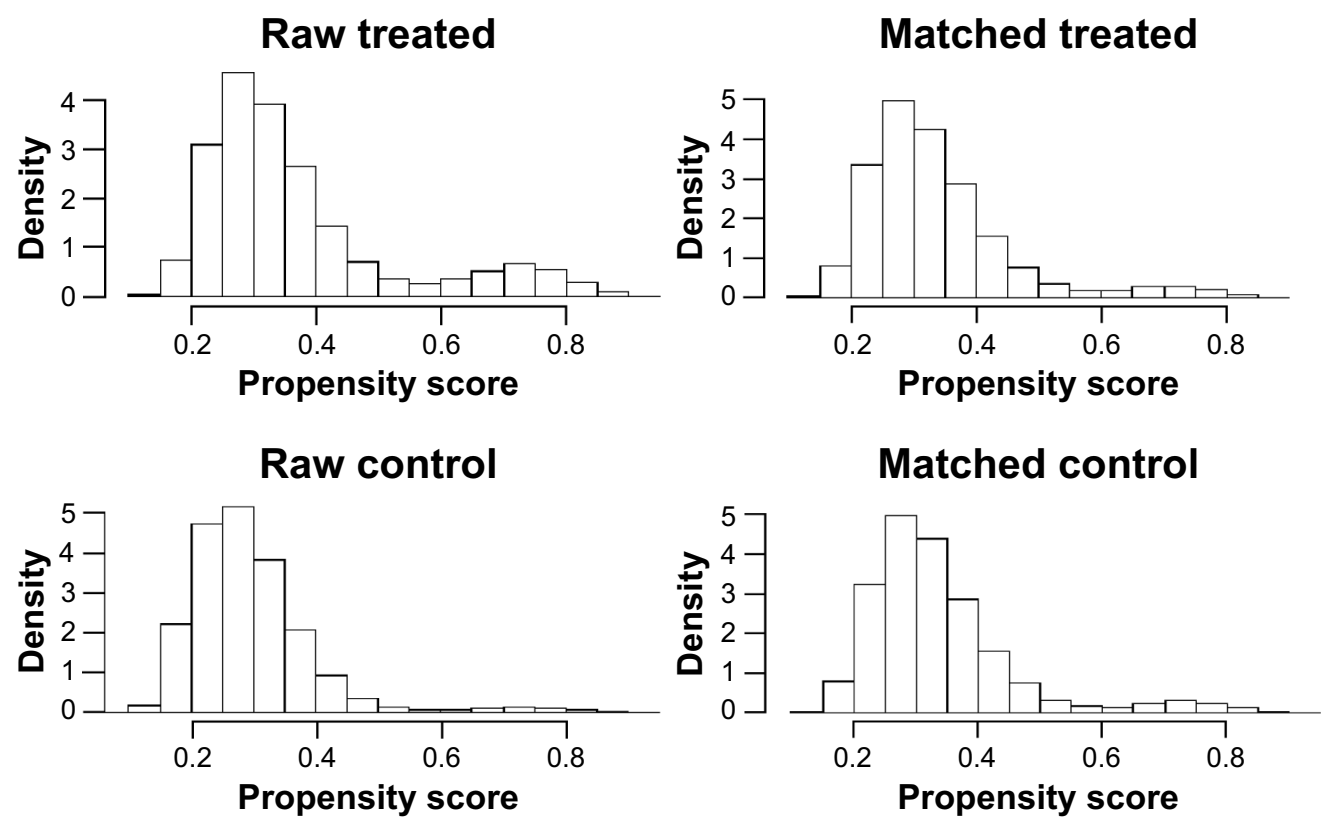

Figure 2 Histograms of the density of propensity scores for cohort before and after matching.

NOD compared to nonusers in patients with hypertension. These results are consistent with the findings of previously performed studies. For the Chinese population, 2 Taiwanese studies have shown that statin use was significantly associated with $15 \%$ and $27 \%$ increase in risk of incident diabetes, respectively. ${ }^{28,29}$ Our results are similar to the one reported in a British study (HR: 1.57, 95\% CI: 1.54-1.59), which was based on the Clinical Practice Research Datalink. ${ }^{30}$ However, the risk seems even higher in two Korean studies (HR: 1.84, 95\% CI: 1.63-2.09; HR: 1.99, 95\% CI: 1.36-2.92). ${ }^{31,32}$ Compared to the results of these observational studies, we observed a moderately increased risk of NOD in statin users. On the other hand, the incidence of NOD was higher in our study than that in previous studies. One potential reason for this higher incidence could be that our study subjects were patients with hypertension who were at higher risk for incident diabetes than the general population. ${ }^{28,30}$

Since this was an observational study, the way we defined the cohort may induce bias. When researchers attempt to use a simple cohort approach to emulate the randomized controlled trial design, a form of bias called immortal time bias may be produced. ${ }^{33}$ Immortal time bias has been previously described, and several solutions have been proposed to prevent such bias including prescription time-distribution matching and time-dependent analysis. ${ }^{24}$

In our study, we used prescription time-distribution matching method, which means the overall distribution of index date of the nonusers is matched to the users' index date (date of first statin prescription). ${ }^{33}$ The advantage of this design method is that it avoids the imbalance of the index date distribution between the 2 groups without apparent loss of study efficiency. With the balanced index date between 2 groups, we could better define the lookback period to identify the comorbidities and concomitant medicine intake.

We also conducted the subgroup analysis by sex, age, BMI, statin types, and statin potency. In multivariate Cox model, statin use was associated with increased risk of NOD in males (adjusted HR: 1.58, 95\% CI: 1.42-1.77) and females (adjusted HR: 1.52, 95\% CI: 1.38-1.67). A previous study suggested that incident NOD associated with statin use may be more common in women. ${ }^{10}$ We observed consistently increased risk of NOD by statin use across subgroups by age. This association was decreased but remained significant in older patients. As far as we know, there were 2 reports that demonstrated results similar to our finding. ${ }^{28,30}$ Macedo et al ${ }^{30}$ found that incidence rates of T2DM increased with age in nonusers, whereas in statin users incidence rates remained high. One of the potential mechanisms is that younger people at high risk of developing diabetes are accurately targeted for statin treatment, which would moderate the expected age gradient. Besides, our finding showed that patients with a BMI lower than 24 were at greater risk (adjusted HR: 1.65, 95\% CI: 1.50-1.83) for NOD than those with BMI of 28 or higher (adjusted HR: 1.44, 95\% CI: 1.17-1.77), even though a significant association was observed in all strata. Similar results had been reported by a study done in postmenopausal women. ${ }^{10}$ These findings have not been well explained. A further study to verify the weight effect on statin-related NOD is warranted. However, these finding suggested that 
Table 2 Baseline characteristics of patients who developed T2DM

\begin{tabular}{|c|c|c|c|}
\hline Characteristics & Non-T2DM & T2DM & $P$-value \\
\hline $\mathrm{N}(\%)$ & $63,821(100)$ & $4,172(100)$ & \\
\hline \multicolumn{4}{|l|}{ Age } \\
\hline Mean \pm SD & $59.63(12.63)$ & $60.81(10.76)$ & $<0.001$ \\
\hline Median (QI-Q3) & $59.00(5 I-68)$ & $61.00(54-68)$ & $<0.00$ I \\
\hline Sex (female) & $34,124(53.5)$ & $2,352(56.4)$ & $<0.001$ \\
\hline Dyslipidemia & $3,346(5.2)$ & $473(11.3)$ & $<0.001$ \\
\hline MI & $36(0.1)$ & $5(0.1)$ & 0.196 \\
\hline CAD & $2,274(3.6)$ & $289(6.9)$ & $<0.001$ \\
\hline CVAs & $834(1.3)$ & $\mathrm{II} 2(2.7)$ & $<0.001$ \\
\hline Heart failure & $758(1.2)$ & $97(2.3)$ & $<0.00$ I \\
\hline Angina pectoris & $811(1.3)$ & $85(2.0)$ & $<0.001$ \\
\hline \multicolumn{4}{|l|}{$\mathrm{BMI}, \mathrm{kg} / \mathrm{m}^{2}$} \\
\hline Mean \pm SD & $23.45(4.93)$ & $24.30(3.27)$ & $<0.001$ \\
\hline Median (QI-Q3) & $23.20(21.50-25.10)$ & $24.00(22.20-26.00)$ & \\
\hline $\mathrm{BMI}, \mathrm{kg} / \mathrm{m}^{2}$ & & & $<0.001$ \\
\hline$<24$ & $39,283(61.6)$ & $2,051(49.2)$ & \\
\hline $24-28$ & $4,173(6.5)$ & $499(12.0)$ & \\
\hline$\geq 28$ & 20,365 (31.9) & I,622 (38.9) & \\
\hline Cigarette smoking & & & $<0.00$ I \\
\hline Never & $48,064(75.3)$ & $3,156(75.6)$ & \\
\hline Past & $5,946(9.3)$ & $478(11.5)$ & \\
\hline Current & $9,811(15.4)$ & $538(12.9)$ & \\
\hline \multicolumn{2}{|l|}{ Alcohol consumption } & & 0.359 \\
\hline Never & $50,039(78.4)$ & $3,234(77.5)$ & \\
\hline Occasional & $3,65 \mid(5.7)$ & $255(6.1)$ & \\
\hline Often & $10,13 \mid(15.9)$ & $683(16.4)$ & \\
\hline Physical activity & & & $<0.001$ \\
\hline$<\mathrm{ld} / \mathrm{wk}$ & $28,137(44.1)$ & $2,018(48.4)$ & \\
\hline $\mathrm{I}-4 \mathrm{~d} / \mathrm{wk}$ & $21,338(33.4)$ & $1,416(33.9)$ & \\
\hline$>4 \mathrm{~d} / \mathrm{wk}$ & $14,346(22.5)$ & $738(17.7)$ & \\
\hline$\beta$ blockers & $9,101(14.3)$ & $578(13.9)$ & $0.48 I$ \\
\hline Diuretics & $12,727(19.9)$ & $909(21.8)$ & 0.004 \\
\hline ACEI & $14,959(23.4)$ & I,098 (26.3) & $<0.001$ \\
\hline ARBs & $10,344(16.2)$ & $819(19.6)$ & $<0.001$ \\
\hline $\mathrm{CCBs}$ & $8,163(12.8)$ & $627(15.0)$ & $<0.001$ \\
\hline Type of statin & & & $<0.00$ I \\
\hline Non-statin & $44,226(69.3)$ & $2,216(53.1)$ & \\
\hline Atorvastatin & $8,553(13.4)$ & $710(17.0)$ & \\
\hline Fluvastatin & $153(0.2)$ & $17(0.4)$ & \\
\hline Rosuvastatin & $\mathrm{I}, 32 \mathrm{I}(2 . \mathrm{I})$ & $82(2.0)$ & \\
\hline Simvastatin & $9,568(15.0)$ & $\mathrm{I}, \mathrm{I} 47(27.5)$ & \\
\hline
\end{tabular}

Note: Unless indicated all values in this table are presented as $n(\%)$.

Abbreviations: T2DM, type 2 diabetes mellitus; Ml, myocardial infarction; CAD, coronary artery disease; CVAs, cerebrovascular accidents; BMI, body mass index; d, day; wk, week; ACEl, angiotensin-converting enzyme inhibitors; ARBs, angiotensin receptor blockers; CCBs, calcium channel blockers. we should pay more attention to the prescription of statins to younger patients with normal BMI.

Many studies have investigated the impact of specific statins on the incidence of NOD and have suggested that atorvastatin and simvastatin are more prone to be associated with increased risk of NOD. ${ }^{10,31,32}$ Our findings seemed to be consistent with these findings. The results of the multivariate Cox models demonstrated that atorvastatin users and simvastatin users had the highest risk of NOD. Due to the fact that only a few patients were treated with fluvastatin and rosuvastatin, the $95 \%$ CI of HR for these two subgroups were wider. The multivariate adjusted HRs were 1.44 (95\% CI: 0.83-2.48) and 1.47 (95\% CI: 1.19-1.83), respectively. Some studies have reported that statin-related NOD risk is likely potency dependent. ${ }^{34,35}$ We found that risk for NOD increased with increasing intensity of statins in our study. Compared to nonusers, the adjusted HRs were 1.44 (95\% CI: 1.29-1.61), 1.65 (95\% CI: 1.53-1.79), and 1.80 (95\% CI: 0.86-2.82) for low-, moderate-, and high-potency statin users, respectively.

\section{Limitations and strengths}

Our results should be interpreted cautiously because of some limitations. First, we identified the patients with hypertension, diabetes mellitus, or other comorbidities by using the inpatient and outpatient diagnosis on ICD-10 codes. Our T2DM outcome definition, consisting of a diagnosis of T2DM or a prescription for a diabetes medication in outpatient and inpatient records, was probably unable to capture the actual diabetic patients. However, most of the observational studies based on the existing database suffered the same problems. ${ }^{34,36}$ Second, there were some confounding factors that we could not control. The information about the levels of dyslipidemia and hypertension was lacking, which were potentially important risk factors for the initiation of T2DM. ${ }^{17,18,37}$ Besides, dietary patterns are

Table 3 Incidence rates of NOD by statin exposure status

\begin{tabular}{|c|c|c|c|c|c|}
\hline Group & $\begin{array}{l}\text { Number } \\
\text { of patients }\end{array}$ & $\begin{array}{l}\text { Number } \\
\text { of outcome }\end{array}$ & $\begin{array}{l}\text { Total } \\
\text { person-years }\end{array}$ & $\begin{array}{l}\text { Incidence outcome } \\
\text { per I,000 PYs }\end{array}$ & $\begin{array}{l}\text { Adjusted HR } \\
\text { (95\%) }\end{array}$ \\
\hline \multicolumn{6}{|l|}{ NOD after I 80 days } \\
\hline Non & 46,442 & $\mathrm{I}, 884$ & $132,767.5$ & 14.19 & $1.55(1.44-1.66)$ \\
\hline Statin & $21,55 \mid$ & $\mathrm{I}, 537$ & $59,848.48$ & 25.68 & \\
\hline Non (matched) & 19,818 & 917 & $56,445.33$ & 16.25 & I.54 (I.4I-I.67) \\
\hline Statin (matched) & 19,818 & $\mathrm{I}, 334$ & $55,237.63$ & 24.15 & \\
\hline \multicolumn{6}{|l|}{ All NOD } \\
\hline Non & 46,442 & 2,216 & $132,767.5$ & 16.69 & $1.75(1.64-1.87)$ \\
\hline Statin & 21,551 & 1,956 & $59,848.48$ & 32.68 & \\
\hline Non (matched) & 19,818 & $\mathrm{I}, 079$ & $56,445.33$ & 19.06 & $1.65(1.53-1.78)$ \\
\hline Statin (matched) & 19,818 & $\mathrm{I}, 729$ & $55,237.63$ & 31.31 & \\
\hline
\end{tabular}

Abbreviations: NOD, now onset of diabetes mellitus; HR, hazard ratio. 


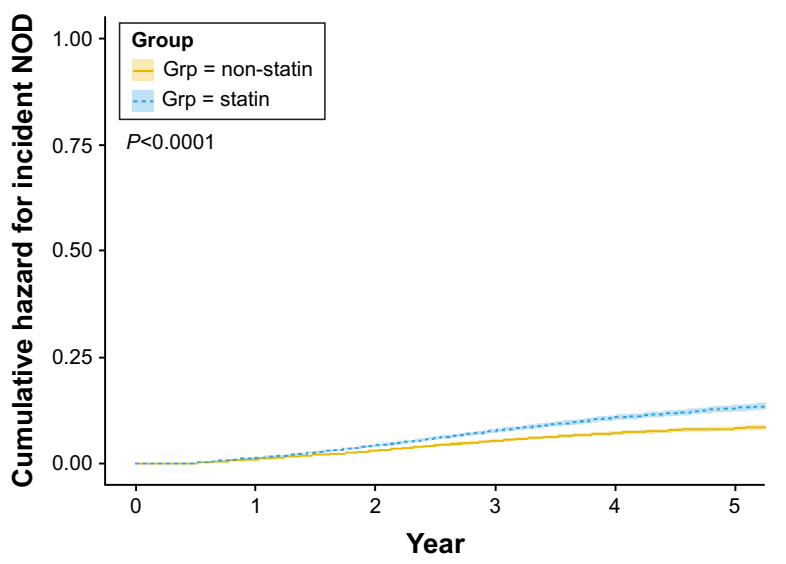

Figure 3 Cumulative incidence of NOD between new users of statin and non-statin users during the follow-up period.

Abbreviations: NOD, new-onset of diabetes mellitus; grp, group. crucial determinants of $\mathrm{T} 2 \mathrm{DM},{ }^{38}$ and data regarding this were not collected in the Yinzhou database. This might be the reason that we found relatively high HRs in our study. Third, we could only identify if the doctors prescribed drugs for the patients from the database; we have no idea whether the patients actually took the medications.

Our study also had some strengths. First, our study was the first to investigate the risk of statins in hypertensive population of Chinese mainland by using a well-developed health care database which reflects the physician behavior in a real-life setting. Second, we conducted a new users and new-onset T2DM design by excluding the patients who had took statins or had already been being diagnosed as having T2DM. Third, we use prescription time-distribution

Table 4 Risk for incident NOD by statin use, within sex, age, BMI, smoke status, alcohol use, exercise frequency, statin types, and potency subgroups

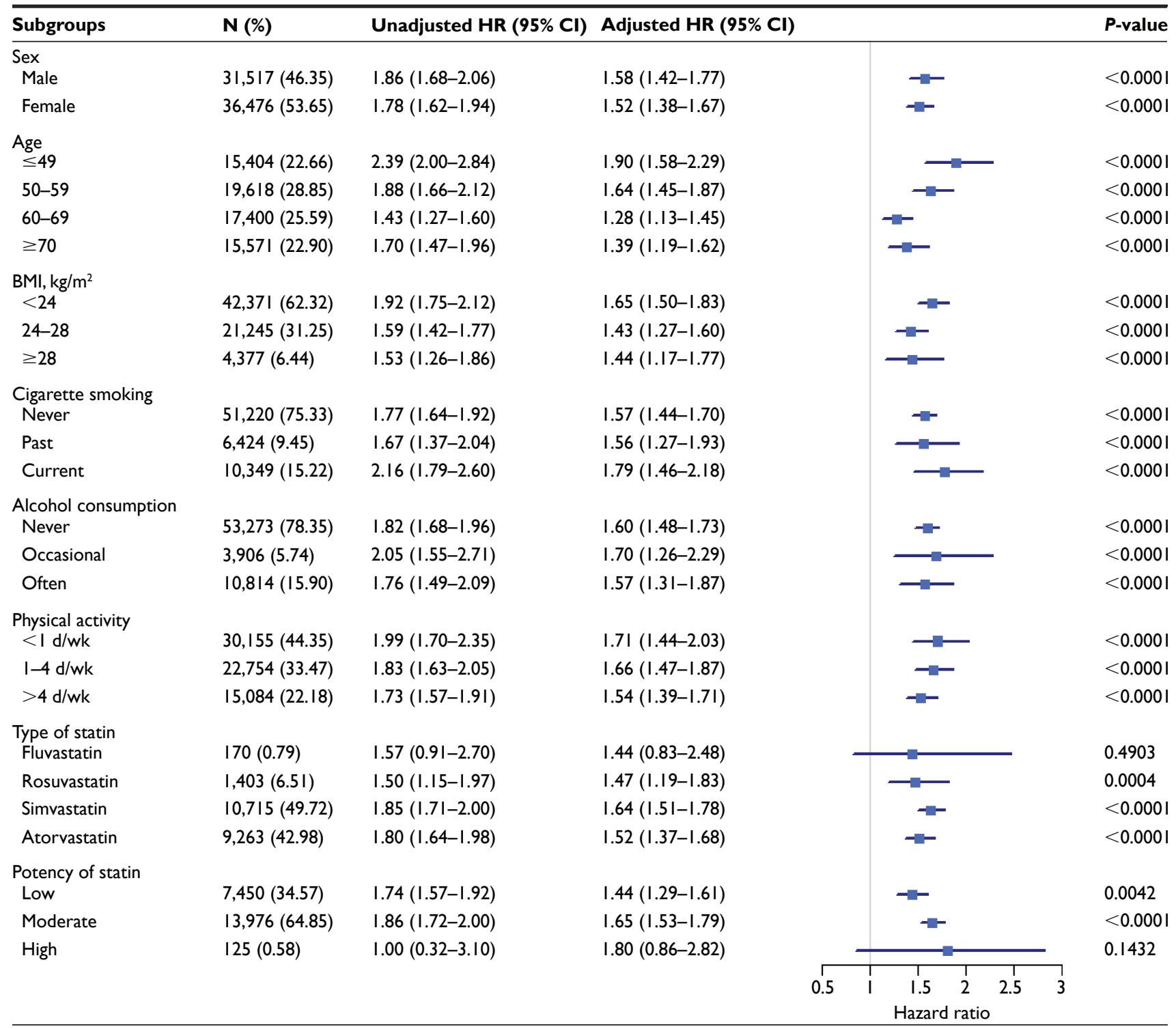

Abbreviations: NOD, new-onset diabetes mellitus; HR, hazard ratio; Cl, confidence interval; BMI, body mass index; d, day; wk, week. 
matching method to avoid immortal time bias and better identify the baseline characteristics. Forth, compared to other Asian studies, ${ }^{28,29,31,32}$ some crucial confounders (smoking status, alcohol use, and exercise frequency) were included in our analysis.

\section{Conclusion}

Our findings indicated that statin therapy was associated with an increased risk for incident NOD compared to nonusers of statins among hypertensive patients. Regardless of patients' characteristics, statin types, or dosage, the association remained. The risk seems to be smaller among old and overweight patients who are at higher risk for CVD. These data provide better evidence about the association between statins and NOD in a real-world context in mainland People's Republic of China. As the benefits of statins overwhelm the risk, especially for patients who are in a higher risk for CVD or with existing CVD, clinicians should followed the guideline to consider the statin therapy. As for those younger than 50 years old, the tradeoff between statin's benefits and adverse effects should be well discussed. These findings should be interpreted cautiously as observational studies are subject to bias and residual confounding that cannot be ruled out.

\section{Acknowledgment}

This work was supported by the National Natural Science Foundation of China (No. 91646107). We thank Prof Dorothee Bartels for reviewing the article and giving suggestions about the study design.

\section{Author contributions}

Hailong Li, Hongbo Lin, and Siyan Zhan contributed to the study concept and design and take responsibility for the integrity of the data and the accuracy of the data analysis. Hailong Li, Houyu Zhao, Yingchu Cheng, Yang Xu, and Peng Shen obtained and conducted statistical analysis. Hailong Li drafted the report, which was edited by Siyan Zhan. All authors contributed toward data analysis, drafting and critically revising the paper and agree to be accountable for all aspects of the work. All authors have reviewed and approved the final version of manuscript.

\section{Disclosure}

The authors would like to declare that the abstract of this article was presented at the 33rd International Conference on Pharmacoepidemiology \& Therapeutic Risk Management (Montreal, August 26-30, 2017) and 10th Asian Conference on Pharmacoepidemiology (Brisbane, October 29-31, 2017). It was published in Pharmacoepidemiology \& Drug Safety (Volume 26, Issue S2, August 2017). The authors report no other conflicts of interest in this work.

\section{References}

1. Baigent C, Keech A, Kearney PM, et al. Efficacy and safety of cholesterol-lowering treatment: prospective meta-analysis of data from 90,056 participants in 14 randomised trials of statins. Lancet. 2005; 366(9493):1267-1278.

2. Baigent C, Blackwell L, Emberson J, et al; Cholesterol Treatment Trialists' (CTT) Collaboration. Efficacy and safety of more intensive lowering of LDL cholesterol: a meta-analysis of data from 170,000 participants in 26 randomised trials. Lancet. 2010;376(9753):1670-1681.

3. Brugts JJ, Yetgin T, Hoeks SE, et al. The benefits of statins in people without established cardiovascular disease but with cardiovascular risk factors: meta-analysis of randomised controlled trials. BMJ. 2009; 339:b2376.

4. Rajpathak SN, Kumbhani DJ, Crandall J, Barzilai N, Alderman M, Ridker PM. Statin therapy and risk of developing type 2 diabetes: a meta-analysis. Diabetes Care. 2009;32(10):1924-1929.

5. Sattar N, Preiss D, Murray HM, et al. Statins and risk of incident diabetes: a collaborative meta-analysis of randomised statin trials. Lancet. 2010;375(9716):735-742.

6. Koh KK, Quon MJ, Han SH, Lee Y, Kim SJ, Shin EK. Atorvastatin causes insulin resistance and increases ambient glycemia in hypercholesterolemic patients. J Am Coll Cardiol. 2010;55(12):1209-1216.

7. Sabatine MS, Wiviott SD, Morrow DA, McCabe CH, Cannon CP. Highdose atorvastatin associated with worse glycemic control: A PROVE-IT TIMI 22 substudy. Circulation. 2004;110(17):834.

8. Thongtang N, Ai M, Otokozawa S, et al. Effects of maximal atorvastatin and rosuvastatin treatment on markers of glucose homeostasis and inflammation. Am J Cardiol. 2011;107(3):387-392.

9. Ridker PM, Danielson E, Fonseca FAH, et al. Rosuvastatin to prevent vascular events in men and women with elevated C-reactive protein. New Engl J Med. 2008;359(21):2195-2207.

10. Culver AL, Ockene IS, Balasubramanian R, et al. Statin use and risk of diabetes mellitus in postmenopausal women in the Women's Health Initiative. Arch Intern Med. 2012;172(2):144-152.

11. Sukhija R, Prayaga S, Marashdeh M, et al. Effect of statins on fasting plasma glucose in diabetic and nondiabetic patients. $J$ Investig Med. 2009;57(3):495-499.

12. Wang P. Statin dose in Asians: is pharmacogenetics relevant? Pharmacogenomics. 2011;12(11):1605-1615.

13. Liao JK. Safety and efficacy of statins in Asians. Am J Cardiol. 2007; 99(3):410-414.

14. Lee E, Ryan S, Birmingham B, et al. Rosuvastatin pharmacokinetics and pharmacogenetics in white and Asian subjects residing in the same environment. Clin Pharmacol Ther. 2005;78(4):330-341.

15. Sun YL, Gregersen H, Yuan W. Chinese health care system and clinical epidemiology. Clin Epidemiol. 2017;9:167-178.

16. Hu DY. New guidelines and evidence for prevention and treatment of dyslipidemia and atherosclerotic cardiovascular disease in China. Chronic Dis Transl Med. 2017;3(2):73-74.

17. Izzo R, de Simone G, Chinali M, et al. Insufficient control of blood pressure and incident diabetes. Diabetes Care. 2009;32(5):845-850.

18. Gress TW, Nieto FJ, Shahar E, Wofford MR, Brancati FL. Hypertension and antihypertensive therapy as risk factors for type 2 diabetes mellitus. Atherosclerosis Risk in Communities Study. New Engl J Med. 2000; 342(13):905-912.

19. Alderman MH, Cohen H, Madhavan S. Diabetes and cardiovascular events in hypertensive patients. Hypertension. 1999;33(5): $1130-1134$.

20. Sowers JR, Epstein M, Frohlich ED. Diabetes, hypertension, and cardiovascular disease: an update. Hypertension. 2001;37(4):1053-1059. 
21. Yusuf S, Bosch J, Dagenais G, et al. Cholesterol lowering in intermediate-risk persons without cardiovascular disease. New Engl J Med. 2016;374(21):2021-2031.

22. Morales Salinas A, Coca A, Olsen MH, et al. Clinical perspective on antihypertensive drug treatment in adults with grade 1 hypertension and low-to-moderate cardiovascular risk: an international expert consultation. Curr Probl Cardiol. 2017;42(7):198-225.

23. Mancia G, Fagard R, Narkiewicz K, et al. 2013 ESH/ESC Guidelines for the management of arterial hypertension: the Task Force for the management of arterial hypertension of the European Society of Hypertension $(\mathrm{ESH})$ and of the European Society of Cardiology (ESC). J Hypertens. 2013;31(7):1281-1357.

24. Zhou Z, Rahme E, Abrahamowicz M, Pilote L. Survival bias associated with time-to-treatment initiation in drug effectiveness evaluation: a comparison of methods. Am J Epidemiol. 2005;162(10): 1016-1023.

25. Wang JB, Gu MJ, Shen P, et al. Body mass index and mortality: a 10-year prospective study in China. Sci Rep. 2016;6:31609.

26. Zhao M, Lin H, Yuan Y, et al. Prevalence of pre-diabetes and its associated risk factors in rural areas of Ningbo, China. Int $J$ Environ Res Public Health. 2016;13(8):pii: E808.

27. Yang Y, Zhou X, Gao S, et al. Evaluation of electronic healthcare databases for post-marketing drug safety surveillance and pharmacoepidemiology in China. Drug safety. 2017;41(1):125-137.

28. Wang KL, Liu CJ, Chao TF, et al. Statins, risk of diabetes, and implications on outcomes in the general population. J Am Coll Cardiol. 2012; 60(14):1231-1238.

29. Lin ZF, Wang CY, Shen LJ, Hsiao FY, Wu FLL. Statin use and the risk for incident diabetes mellitus in patients with acute coronary syndrome after percutaneous coronary intervention: a population-based retrospective cohort study in Taiwan. Can J Diabetes. 2016;40(3): 264-269.
30. Macedo AF, Douglas I, Smeeth L, Forbes H, Ebrahim S. Statins and the risk of type 2 diabetes mellitus: cohort study using the UK clinical practice pesearch datalink. BMC Cardiovasc Disord. 2014;14:85.

31. Lee J, Noh Y, Shin S, et al. Impact of statins on risk of new onset diabetes mellitus: a population-based cohort study using the Korean National Health Insurance claims database. Ther Clin Risk Manag. 2016;12:1533-1543.

32. Rha SW, Choi BG, Seo HS, et al. Impact of statin use on development of new-onset diabetes mellitus in Asian population. Am J Cardiol. 2016;117(3):382-387.

33. Suissa S. Immortal time bias in observational studies of drug effects. Pharmacoepidemiol Drug Saf. 2007;16(3):241-249.

34. Dormuth CR, Filion KB, Paterson JM, et al. Higher potency statins and the risk of new diabetes: multicentre, observational study of administrative databases. BMJ. 2014;348:g3244.

35. Preiss D, Seshasai SR, Welsh P, et al. Risk of incident diabetes with intensive-dose compared with moderate-dose statin therapy: a metaanalysis. JAMA. 2011;305(24):2556-2564.

36. Carter AA, Gomes T, Camacho X, Juurlink DN, Shah BR, Mamdani MM. Risk of incident diabetes among patients treated with statins: population based study. BMJ. 2013;346:f2610.

37. He S, Wang S, Chen X, et al. Higher ratio of triglyceride to high-density lipoprotein cholesterol may predispose to diabetes mellitus: 15-year prospective study in a general population. Metabolism. 2012;61(1):30-36.

38. Parillo M, Riccardi G. Diet composition and the risk of type 2 diabetes: epidemiological and clinical evidence. Br J Nutr. 2004;92(1):7-19.
Therapeutics and Clinical Risk Management

\section{Publish your work in this journal}

Therapeutics and Clinical Risk Management is an international, peerreviewed journal of clinical therapeutics and risk management, focusing on concise rapid reporting of clinical studies in all therapeutic areas outcomes, safety, and programs for the effective, safe, and sustained use of medicines. This journal is indexed on PubMed Central, CAS,

\section{Dovepress}

EMBase, Scopus and the Elsevier Bibliographic databases. The manuscript management system is completely online and includes a very quick and fair peer-review system, which is all easy to use. Visit http://www.dovepress.com/testimonials.php to read real quotes from published authors. 